Arch. Tierz., Dummerstorf 45 (2002) 6, 523-534

Aus dem Institut für Tierzucht und Tierverhalten der Bundesforschungsanstalt für Landwirtschaft

MARTIN STEINHARDT und HANS - HERMANN THIELSCHER

\title{
Effekte der Entwicklungsqualität auf die Herzfrequenz, die Aktivitäts- und Ruhezeiten und deren Rhythmizität sowie auf die Wachstumsleistung der Milchrindkälber während der Aufzucht in Gruppenhaltung mit Tränkeautomatenfütterung
}

\begin{abstract}
Summary
Title of the paper: Effects of development quality on heart rate, activity and resting times and their diurnal rhythmicity and on growth of group housed feeder-fed dairy calves

To characterize the rhythmicity of physiological variables in dairy calves of different developmental quality and fitness (groups by hemoglobin content of blood) at early gowth (71 German Holstein Friesian, 6 German Red Pied, 36 male and 41 female) long term heart rate recordings were taken at 5, 15, 40 and 60 days of age using Polar Sport Tester, from which the number and duration of activity (ZDA) and resting times (ZDR) and the total daily activity (GZA) and resting time (GZR) could be established. For these periods characteristic heart rate values were calculated (HFA and HFR) and they were analysed for daytime periods of three hours duration at different life ages. Mean HFA and HFR were significantly different between calves of group HbG1, HbG2 and HbG3 at 5 days and 15 days of age and ZDA was significantly different at 5 and 60 days of age. HF and the increase of HF (HFA-HFR) were significantly smaller at 15 days then at 5 days of age. Mean ZDA and GZA and ZDR were greater and the GZR was smaller at 40 and 60 days then at 5 and 15 days of age. Changes of the variables by 40 and 60 days of life took place with different degrees in calves of the three groups. Deviation of HFA and HFR from the mean of the individual daytime heart rate recording showed a rhythmicity that has been affected by feed access of the calves at the feed supply station. Means of ZDA and ZDR were significantly different between daytime periods of three hours duration at the age points. Results show effects of development quality on physiological variables of calves and on the rhythmicity of the variables and what changes occur with advanced development and adaptation of the animals.
\end{abstract}

Key Words: dairy calves, development quality, characteristic heart rate values, activity and resting times, diurnal rhythm, growth performance

\section{Zusammenfassung}

Um die Rhythmizität physiologischer Variablen von Milchrindkälbern mit einer unterschiedlichen Entwicklungsqualität und Fitness (Gruppen nach dem Hämoglobingehalt des Blutes) im Verlaufe der frühen Wachstumsperiode zu charakterisieren, wurden an Kälbern (71 Deutsche Holstein Friesian, 6 Deutsche Rotbunte, 36 männliche, 41 weibliche) vor (5 LT) und während der Gruppenhaltung mit automatischer Fütterung (15, 40 und 60 LT) Langzeitmessungen der Herzfrequenz (HF) mit dem Polar Sport Tester vorgenommen. Mit Hilfe der HFKurven konnten Anzahl und Dauer von Aktivitäts- (ZDA) und Ruheperioden (ZDR), die tägliche Gesamtzeit der Aktivität (GZA) und der Ruhe (GZR) ermittelt, HF-Kennwerte für diese (HFA und HFR) errechnet und für Tageszeitbereiche von $3 \mathrm{Std}$. an den Alterspunkten analysiert werden. HFA und HFR unterschieden sich zwischen HbG1 bis HbG3 bei 5 LT und 15 LT und ZDA bei 5 und 60 LT signifikant. HF und auch die Steigerung (HFAHFR) war bei 15 LT signifikant kleiner als bei 5 LT. Die mittlere ZDA und GZA, der Quotient GZA/GZR und ZDR waren bei 40 und 60 LT größer und die GZR kleiner als bei 5 und 15 LT. Die Änderungen der Variablen bei 40 LT und 60 LT erfolgten in den Kälbergruppen in unterschiedlichem Maße. Abweichungen der HFA und der HFR vom individuellen Mittelwert der Tagesaufzeichnung wiesen eine Periodik auf, die bei den Kälbergruppen durch die Nahrungsverfügbarkeit der Tiere am Futterautomaten beeinflusst wurde. Die Mittelwerte von ZDA und ZDR waren an den Alterspunkten zwischen den Tageszeitbereichen signifikant verschieden. Die Untersuchungsbefunde zeigen den Einfluss der Entwicklungsqualität auf physiologische Variablen der Kälber und deren Rhythmizität im Verlaufe von Wachstum und Adaptation.

Schlüsselwörter: Milchrindkälber, Entwicklungsqualität, Herzfrequenzkennwerte, Aktivitäts- und Ruhezeiten, diurnaler Rhythmus, Wachstum 
1. Einleitung

Die Entwicklungsqualität der Kälber, gemessen an der Variation physiologischer Variablen, kann nach der Geburt beträchtlich sein (STEINHARDT und THIELSCHER, 2000a, 2002b). Sie ist in Verbindung mit direkten und indirekten Verlusten während der Aufzucht untersucht worden (STEINHARDT und THIELSCHER, 2000b; WILSON et al., 2000). In dieser Hinsicht ist die Rhythmizität der Funktionen und physiologischer Variablen von Nutztieren und deren Änderung im Verlaufe der Aufzucht von Interesse. Sie ist in einem Zusammenhang mit dem Tierbetreuungsaufwand und mit technischen Maßnahmen bei verschiedenen Haltungs- und Tränkesystemen zu sehen. Die mit der Nahrungsaufnahme verbundene Rhythmik physiologischer Variablen während der frühen Wachstumsperiode ist bei Kälbern kaum untersucht worden. Die Food-anticipatory activity (FAA) wird für den Output eines Oscillators gehalten, der durch Ereignisse in Verbindung mit der täglichen Fütterung kontrolliert wird (MISTLBERGER, 1994). Das Modell postuliert einen durch Nahrung kontrollierten Oscillator (food entrainable oscillator, FEO) und einen übergeordneten Licht kontrollierten Oscillator (light entrainable oscillator, LEO), die aneinander gekoppelt sind. Unter Bedingungen einer vorübergehenden räumlich und zeitlich begrenzten Nahrungsverfügbarkeit für die Tiere, wie bei den am Futterautomaten aufgezogenen Milchrindkälbern, könnte sich die Entwicklungsqualität und physische Fitness der Kälber auf die Periodizität physiologischer Variablen und auf die Wachstumsleistung der Tiere auswirken.

Im vorliegenden Untersuchungsansatz wurde die Rhythmizität physiologischer Variablen bei Kälbern mit einem unterschiedlichen Hämoglobingehalt des Blutes (Hb) charakterisiert. Die folgenden Fragen waren von Interesse: (1) Wie ist die Herzfrequenz, die Zeitdauer der Aktivitäts- und Ruheperioden und die Wachstumsleistung bei Kälbern mit unterschiedlichem $\mathrm{Hb}$ nach der Geburt? (2) Beeinflusst die Entwicklungsqualität der Kälber in Beziehung zum Alter die Rhythmik physiologischer Variablen und deren Änderungen im Verlaufe der Aufzucht in Gruppen mit Tränkeautomatenfütterung? (3) Welche Änderungen sind an der Aktivitätsperiodik und der HF mit zunehmendem Alter und der Adaptation der Tiere festzustellen?

2.

Material und Methodik

Untersuchte Tiere, Haltungsbedingungen: Kälber der Milchrindherde des Institutes für Tierzucht und Tierverhalten (71 Deutsche Holstein Friesian, DHF; 6 Deutsche Rotbunte, DRB; 36 männliche und 41 weibliche) der Kalbeperiode 1997/1998 wurden untersucht (Tab. 1). Die Haltungsbedingungen der Milchrindkälber sind so, wie sie in einem vorangegangenen Untersuchungsbericht beschrieben worden sind (STEINHARDT und THIELSCHER, 2002a).

Tabelle 1

Übersicht zum Tiermaterial (Characteristics of the calves)

\begin{tabular}{|c|c|c|c|c|}
\hline & Hb-Gruppe 1 & Hb-Gruppe 2 & Hb-Gruppe 3 & Summe \\
\hline Rasse Deutsche Rotbunte & 1 & 2 & 3 & 6 \\
\hline Deutsche Holstein Friesian & 18 & 24 & 29 & 71 \\
\hline Summe & 19 & 26 & 32 & 77 \\
\hline Geschlecht männlich & 9 & 14 & 13 & 36 \\
\hline weiblich & 10 & 12 & 19 & 41 \\
\hline Summe & 19 & 26 & 32 & 77 \\
\hline
\end{tabular}


Untersuchungsablauf, Messungen: HF wurde mit dem Polar Sport Tester (Herzfrequenz Computer von Polar Electro OY, Kempele, Finnland) festgestellt. Das Gerät besteht aus 3 Teilen: Empfänger, Sender und Brustgurt. Die Aufzeichnung der HF erfolgte in 60 sec-Intervallen über 24 Std. Die HF-Kurven wurden mit einem speziellen Programm bearbeitet und für die Aktivitäts- und Ruheperioden im Tagesverlauf HFKennwerte (HFA und HFR) errechnet. Für jedes Tier ist ein Mittelwert der Messung für HFA und HFR ermittelt worden. Die HF-Werte der Aktivitäts- und Ruheperioden im Tagesverlauf ließen sich als Abweichungen von den individuellen Mittelwerten der Messung für HFA bzw. HFR (DIHFA und DIHFR) darstellen, um die Periodik erkennbar zu machen. Aus den HF-Kurven konnten außerdem Beginn und Dauer der Aktivitäts- (ZDA) und der Ruheperioden (ZDR) und die gesamte tägliche Aktivitäts(GZA) und Ruhezeit (GZR) ermittelt werden. Beginn und Dauer der Aktivitäts- und Ruheperioden sind 8 Tageszeitbereichen (TZB) von jeweils 3 Std. zugeordnet worden, die die Grundlage für Berechnungen waren (Abb. 4 bis 7). Durch permanente Videoaufzeichnungen konnten die mit Hilfe der Herzfrequenzkurve ermittelten Aktivitätsund Ruheperioden der Tiere verifiziert werden. Mit Hilfe der Hämoglobinkonzentration des Blutes $(\mathrm{Hb})$ bei $5 \mathrm{LT}$ ist eine Klassifizierung der Kälber vorgenommen worden: HbG1 = Hb <9,2 g/dl; HbG2 = Hb 9,2 bis 10,9 g/dl und HbG3 = Hb $\geq 11$ g/dl.

Bearbeitung der Ergebnisse, statistische Methoden: Die Auswertung fand unter folgenden Gesichtspunkten statt: (1) Prüfung der HF und der ZDA und ZDR sowie GZA und GZR in den Kälbergruppen zwischen den Alterspunkten, (2) Prüfung der Beziehungen von HFA und HFR in den Tageszeitbereichen, an den Alterspunkten und zwischen den Alterspunkten, (3) Prüfung der HF, der Steigerung von HF (HFA-HFR) und der Abweichungen der HF vom individuellen Mittelwert der Tagesaufzeichnungen sowie der ZDA und ZDR zwischen den Tageszeitbereichen in den Hb-Gruppen an den Alterspunkten, (4) Prüfung der Beziehung von GZA und GZR in den Hb-Gruppen an den Alterspunkten. Die Bearbeitung der Ergebnisse wurde mit PC-Statistik von Topsoft Hannover, mit Systat sowie Sigma Plot von SPSS Science Software vorgenommen und die Korrelations- und Regressionsrechnung sowie die Varianzanalyse (ANOVA und ANOVA for repeated measures) angewandt. Mittelwertprüfungen zweier Gruppen wurden mit dem t-Test und Wilcoxon-Test vorgenommen. Die Irrtumswahrscheinlichkeiten (Bonferroni adjusted probability) sind in den Tabellen und Abbildungen angegeben und, wenn nicht ausdrücklich erwähnt, mit $5 \%$ angenommen worden.

\section{Ergebnisse}

\section{Körpermasse, Wachstum}

Das mittlere Geburtsgewicht der Kälber war zwischen den Gruppen nicht signifikant verschieden (Tab. 2). Bei einem Alter von 7 LT war eine große Variation der Wachstumsrate festzustellen. Die mittlere Wachstumsrate war bei HbG1 am kleinsten und bei HbG3 am größten. Bei 50 LT waren die Mittelwertunterschiede der Körpermasse ( $\mathrm{p}=$ 0,0356) und der Wachstumsrate $(p=0,0187)$ zwischen HbG1 und HbG3 signifikant. Bei 78 LT unterschieden sich die Mittelwerte der Körpermasse und der Wachstumsrate nicht signifikant. Die Variation der Körpermasse und der Wachstumsrate der Kälber nahm bis zum Alter von 50 LT in den drei Gruppen in annähernd gleichem 
Maße zu, bis zum Alter von 78 LT nahm sie bei den Tieren in HbG2 und HbG3 stärker zu als bei jenen in HbG1.

Tabelle 2

Körpermasse und Wachstumsrate der Milchrindkälber an verschiedenen Alterspunkten, Mittelwerte und Standardabweichungen (Body weight and growth rate of dairy calves at different life ages, Mean values and Standard deviation)

\begin{tabular}{lcllll}
\hline & & Geburt & 7 LT & 50 LT & 78 LT \\
\hline $\begin{array}{l}\text { Körpermasse Hb-Gruppe 1 } \\
\text { (kg) }\end{array}$ & & 43,7 & 45,6 & $66,2^{\mathrm{a}}$ & 92,3 \\
& Hb-Gruppe 2 & 4,4 & 5,1 & 6,1 & 8,8 \\
& & 43,3 & 45,6 & $68,0^{\mathrm{a}}$ & 94,2 \\
& Hb-Gruppe 3 & 5,0 & 4,7 & 7,0 & 10,9 \\
& & 44,1 & 47,9 & $70,9^{\mathrm{b}}$ & 95,1 \\
Tägliche & Hb-Gruppe 1 & 3,5 & 4,0 & 8,9 & 11,1 \\
Zunahme & & & 0,22 & $0,45^{\mathrm{a}}$ & 0,63 \\
(kg/d) & Hb-Gruppe 2 & & 0,38 & 0,07 & 0,07 \\
& & & 0,3 & $0,48^{\mathrm{a}}$ & 0,65 \\
& Hb-Gruppe 3 & & 0,41 & 0,07 & 0,11 \\
& & & 0,5 & $0,53^{\mathrm{b}}$ & 0,65 \\
\hline
\end{tabular}

LT = Lebenstage; a,b Mittelwerte in der Spalte mit gleichem Buchstaben nicht signifikant verschieden
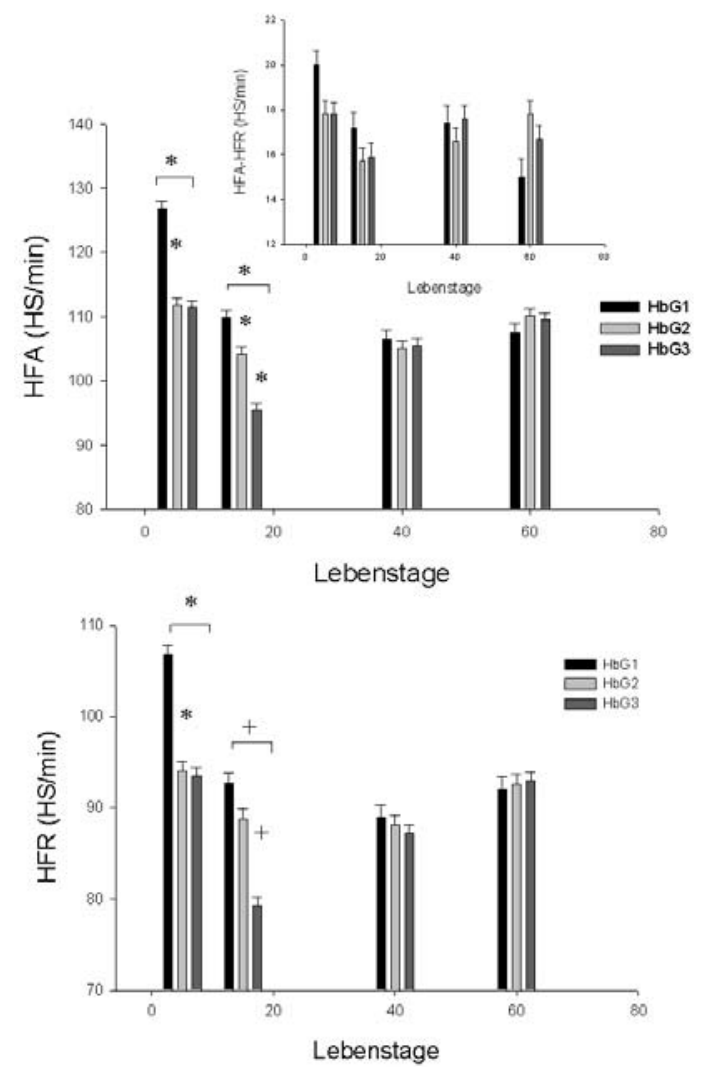

Abb. 1: Herzfrequenz der Aktivitäts- (oberer Teil) und der Ruheperioden (unterer Teil) und Steigerung der Herzfrequenz (Insert) von Milchrindkälbern an 4 Alterspunkten, Gruppen nach dem Hämoglobingehalt des Blutes, Least Squares Means and SEM, $+\mathrm{p}<0,05 ;{ }^{*} \mathrm{p}<0,01$ Mittelwerte zwischen den Gruppen signifikant verschieden (Heart rate of activity (top) and resting times (bottom) and increase in heart rate (insert) in dairy calves at four life ages, groups by hemoglobin content of blood, LSM and SEM; $+\mathrm{p}<0,05,{ }^{*} \mathrm{p}<0,01$ mean values different between groups) 


\section{Herzfrequenz, Aktivitäts- und Ruhezeiten der Milchrindkälber an verschiedenen Alterspunkten}

HFA und HFR änderten sich bis zum Alter der Kälber von 60 LT signifikant (Abb. 1). Bei der Steigerung der HF (HFA-HFR) unterschieden sich nur die Mittelwerte von HbG1 bei 5 LT gegen HbG1 bei 60 LT, gegen HbG2 bei 15 LT und HbG2 bei 40 LT sowie gegen HbG3 bei 15 LT und HbG3 bei 60 LT. HFA und HFR unterschieden sich an den Alterspunkten und in den Gruppen HbG1 bis HbG3 stets signifikant und wiesen enge Korrelationen auf $(\mathrm{p}<0,001)$. Kälber der HbG1 hatten größere HFA und HFR und auch größere Steigerungen der HF (HFA-HFR) bei 5 und 15 LT als jene der HbG2 und HbG3 (Abb. 1).

Ein Einfluss des Geschlechtes auf HFA sowie auf die Steigerung HFA-HFR konnte bei Kälbern der HbG2 und HbG3 festgestellt werden. HFA und die Steigerung der HF (HFA-HFR) lagen bei weiblichen Tieren niedriger als bei männlichen Tieren. Unterschiede der Mittelwerte von ZDR und ZDA ließen sich zwischen den Tieren der HbG1 bis HbG3 und zwischen männlichen und weiblichen Tieren nicht sichern. Der mit Hilfe der Covarianzanalyse untersuchte Einfluss des Alters der Kälber auf ZDR, ZDA, HFA, HFR und auf die Steigerung von HF (HFA-HFR) in den Gruppen nach dem $\mathrm{Hb}$ und nach dem Geschlecht des Kalbes war hoch signifkant. Das Geschlecht des Kalbes hatte einen Einfluss auf HFA und auf die Steigerung der Herzfrequenz (HFAHFR), nicht auf HFR.

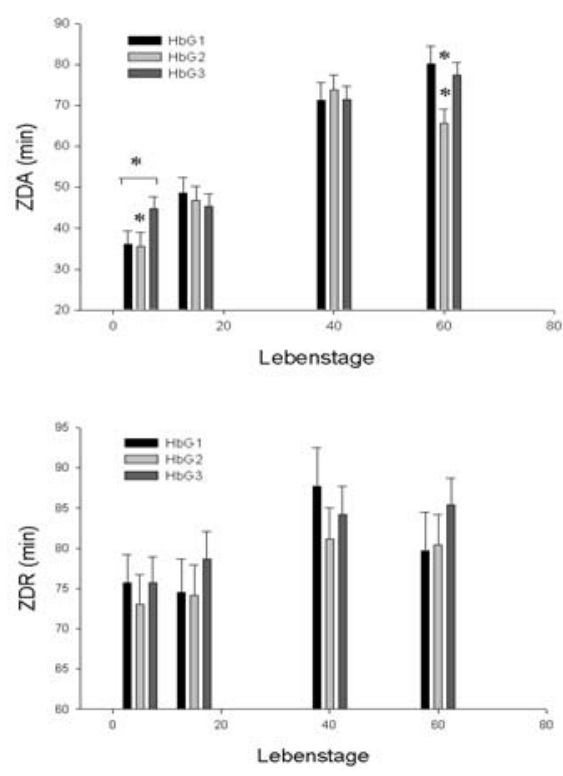

Abb. 2: Zeitdauer der Aktivitäts- (oberer Teil) und der Ruheperioden (unterer Teil) von Milchrindkälbern an 4 Alterspunkten, weiter wie bei Abb. 1 (Duration of activity (top) and of resting times (bottom) in dairy calves at four life ages)

ZDA und insbesondere ZDR wiesen an allen Alterspunkten eine große Variation in allen Gruppen auf (Abb. 2 ). Die Mittelwerte von ZDA unterschieden sich zwischen den Kälbern in HbG1 bis HbG3 an den Alterspunkten 1 und 4 und zwischen den Alterspunkten sicher (Abb. 2), diejenigen von ZDR nicht. Auffällig waren die größeren mittleren ZDA und ZDR der Kälber im Alter von 40 LT und 60 LT gegenüber 5 LT und 15 LT (Abb. 2). Die Mittelwerte von GZA und GZR unterschieden sich zwischen HbG1 bis HbG3 nicht signifikant, zwischen den Alterspunkten jedoch signifikant (Abb. 3). Zwischen GZA und GZR der Kälber ließ sich eine negative Korrelation 
nachweisen, die an den Alterspunkten $40 \mathrm{LT}$ und $60 \mathrm{LT}$ enger (adjusted multiple $\mathrm{r}=$ 0,835 und $\mathrm{r}=0,913$ ) als bei $5 \mathrm{LT}$ und $15 \mathrm{LT}$ (adjusted multiple $\mathrm{r}=0,617$ und $\mathrm{r}=$ $0,463)$ war.
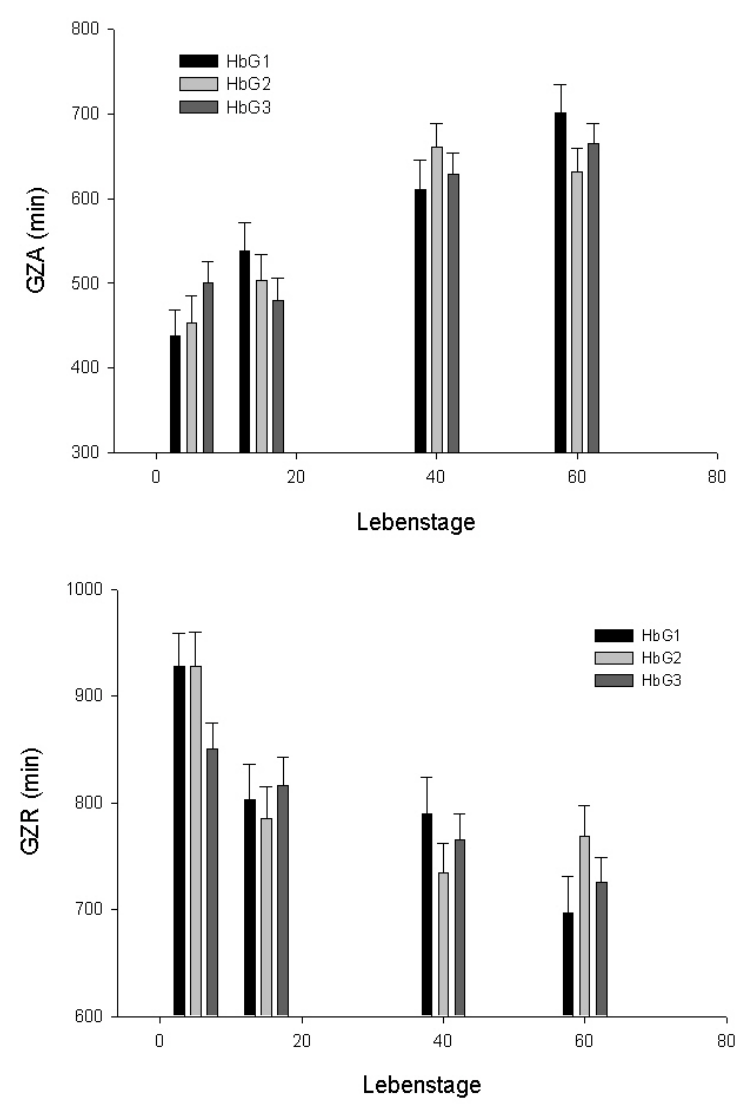

Abb. 3: Tägliche Gesamtzeit der Aktivität (oberer Teil) und der Ruhe (unterer Teil) von Milchrindkälbern an 4 Alterspunkten, weiter wie bei Abb. 1 (Total daily time of activity (top) and of rest (bottom) in dairy calves at four life ages)

\section{Periodik von Herzfrequenz, Aktivität und Ruhe}

Häufungen größere HF und größerer Steigerungen der HF (HFA-HFR) waren an den Tränkepunkten (07.00 und 16.00 Uhr) bei Kälbern im Alter von 5 LT und an den Abrufzeitpunkten des MAT (03.00, 08.00, 15.00 und 20.00 Uhr) bei Kälbern im Alter von 15, 40 und 60 LT sowie in der Servicezeit festzustellen (Ergebnisse nicht weiter angeführt). Signifikante Unterschiede der Mittelwerte der Variablen zwischen den Tageszeitbereichen waren häufiger bei Kälbern von HbG2 und HbG3 als bei jenen von HbG1. Die Abweichungen der HF vom individuellen Mittelwert der Tagesaufzeichnung (DIHFA und DIHFR) ließen die unterschiedliche Anpassung der Kälber und die Periodik der Variablen an den Alterspunkten erkennen (Abb. 4 bis 7). Unterschiede zwischen HbG1, HbG2 und HbG 3 zeichneten sich bis zum Alter der Kälber von 15 LT zwischen 00.00 Uhr und 09.00 Uhr sowie zwischen 18.00 und 24.00 Uhr ab (Abb. 4 und 5). Kälber in HbG1 hatten kleinste HFA zwischen 06.00 und 15.00 Uhr und größte HFA zwischen 21.00 und 03.00 Uhr. Kälber der HbG2 und HbG3 hatten kleinste HFA zwischen 00.00 und 06.00 Uhr sowie zwischen 09.00 und 15.00 Uhr und größte HFA zwischen 18.00 und 24.00 Uhr. Außerdem hatten diese Tiere gegenüber 
jenen von HbG1 eine größere HFA in der Zeit von 06.00 bis 09.00 Uhr. Die Zweigipfligkeit der Kurve bildete sich bei allen Tieren im Verlaufe der Aufzucht heraus, bei den Kälbern der drei Hb-Gruppen jedoch in unterschiedlichem Maße (Abb. 4 und 5). Bemerkenswert waren weiterhin die stärker ausgeprägten Schwingungen der HFA bei den Kälbern in HbG2. Die morgendliche Steigerung der HFA verlagerte sich bei allen Kälbern von 06.00 bis 09.00 Uhr auf 03.00 bis 06.00 Uhr. Die Amplituden der Schwingungen von HFA verringerten sich insbesondere bei Kälbern der HbG3, wenn die Tiere in die benachbarte Entwöhnungsgruppe gekommen waren (Abb. 4 und 5). Die Schwingungen von HFR im Tagesverlauf waren an allen Alterspunkten deutlich, so dass die Mittelwerte der Tageszeitbereiche sich stets signifikant unterschieden. HFR wies bei 5 LT kleinste Werte zwischen 03.00 und $06.00 \mathrm{Uhr}$ und größte zwischen 18.00 und 21.00 Uhr auf (Abb. 6 und 7). Die Amplituden der Schwingungen von HFR im Tagesverlauf wurden bei allen Kälbern größer, und die Minima und Maxima der HFR wurden bei Tieren der HbG1 bis HbG3 in den Tageszeitbereichen in unterschiedlichem Maße verlagert (Abb. 6 und 7).
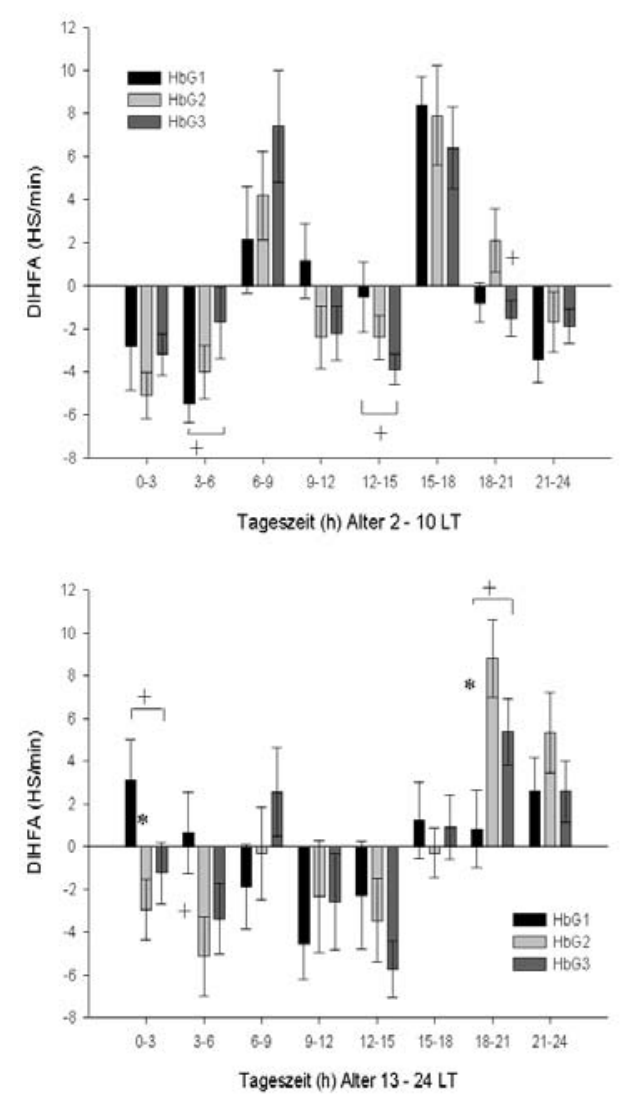

Abb. 4: Abweichung der Herzfrequenz während der Aktivitätsperioden vom individuellen Mittelwert der Tagesaufzeichnung bei einem Alter der Milchrindkälber von 2 bis 10 LT (oberer Teil) und von 13 bis 24 LT (unterer Teil), weiter wie Abb. 1 (Deviation of heart rate from the mean value of individual daily recording at activity times in calves of 2 to 10 days of lif age (top) and of 13 to 24 days of life age (bottom))

\section{4.}

Diskussion

An der großen interindividuellen Variation der HF, die bei mutterlos aufgezogenen Milchrindkälbern (ERMGASSEN, 1996) und auch bei Saugkälbern der Mutterkuhhaltung (LEHR, 1997; STEINHARDT et al., 1997) festgestellt worden ist, ist außer der typmäßigen Reaktionsweise in erheblichem Maße die Entwicklungsqualität der 
Kälber beteiligt. Einflüsse des Geschlechtes des Kalbes auf HF wurden in den beiden ersten Lebenswochen durch solche der Entwicklungsqualität überlagert und waren erst in der folgenden Zeit nachweisbar. Die größere Herzfrequenz und die anfänglich kleinere Wachstumsrate der Kälber von HbG1 stehen ursächlich mit der kleinen Hämoglobinkonzentration des Blutes dieser Tiere in Verbindung. Die Verkleinerung der HF zwischen 5 und 15 LT (ERMGASSEN, 1996) war in der vorliegenden Untersuchung nur bei Kälbern in HbG2 und HbG3 nachweisbar. Dies ist auf die Anpassung der Blutkreislauffunktion und auf die Änderung des Stoffwechsels zurückzuführen. Obwohl sich die Wachstumsrate der Kälber von HbG1 im Verlaufe der Aufzucht vergrößerte und die Differenz der Leistungsmerkmale zwischen den Hb-Gruppen sich verringerte, blieben die Unterschiede zwischen den Körpermassen der Kälber von HbG1 nach HbG3 bestehen (Tab. 2). Bemerkenswert waren die interindividuell unterschiedliche Wachstumsleistung der Kälber und eine zunehmende Variation der Körpermasse im Verlaufe der Aufzucht insbesondere in HbG2 und HbG3.
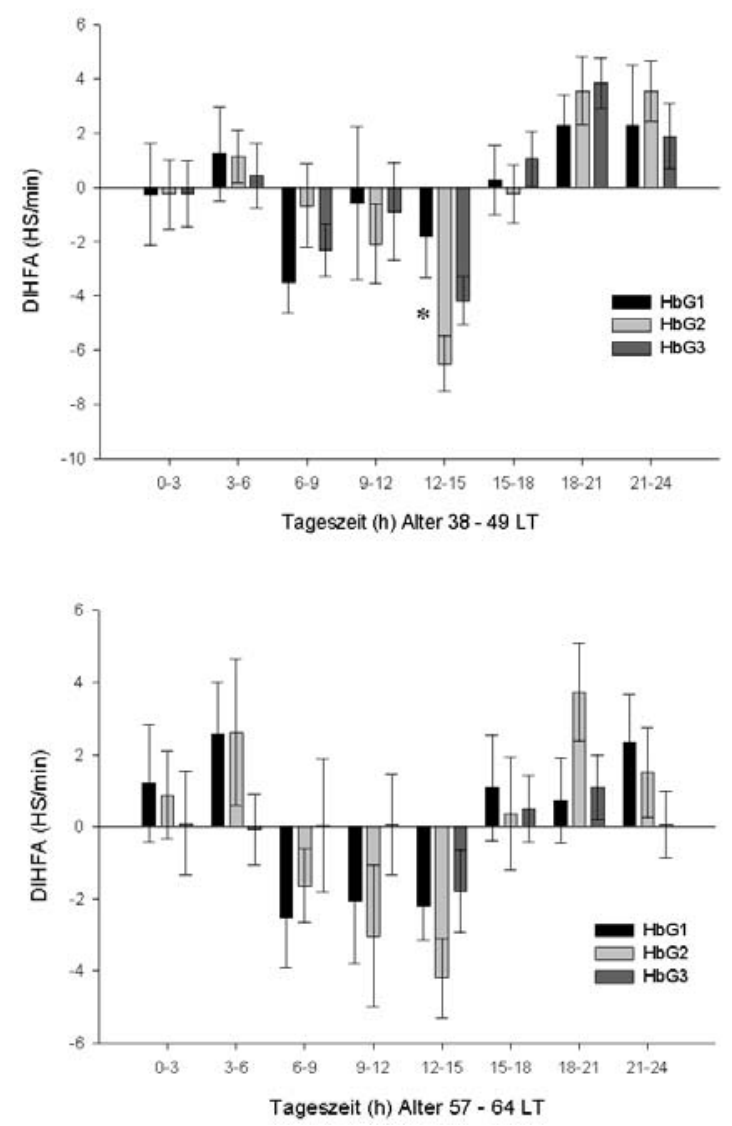

Abb. 5: Abweichung der Herzfrequenz während der Aktivitätsperioden vom individuellen Mittelwert der Tagesaufzeichnung bei einem Alter der Milchrindkälber von 38 bis 49 LT (oberer Teil) und von 57 bis 64 LT (unterer Teil), weiter wie bei Abb. 1 (Deviation of heart rate from the mean value of individual daily recording at activity times in calves of 38 to 49 days of life age (top) and of 57 to 64 days of life age (bottom))

Die bei den Kälbern individuell sehr verschiedene Frequenz und Zeitdauer von Liegen, Stehen und motorischer Aktivität (Abb. 2) und unterschiedliche Änderungen von ZDA und ZDR sowie GZA und GZR der Kälber aus HbG1, HbG2 und HbG3 an den Alterspunkten stehen mit der physischen Fitness und der Nahrungsverfügbarkeit der Tiere in Verbindung. Kompetitive Bedingungen bei der Aufnahme der Flüssignahrung und des Konzentratfutters wurden von den Tieren in unterschiedlicher Weise bewältigt. Mehr- 
fach konnte beobachtet werden, dass einige Tiere die Fähigkeit entwickelten, am Tränkestand und am Kraftfutterautomaten größere als die vorgesehenen Mengen zu erlangen, was mit einem längeren Aufenthalt am Automaten verbunden war. Bei 4 Tränkeabrufterminen waren drei „Aktivitätsphasen“ und ein hohes „Aktivitätsniveau“ zwischen 08.00 und 12.00 Uhr (Servicezeit 09.00 bis $12.00 \mathrm{Uhr}$ ), zwischen 14.00 und 18.00 Uhr sowie zwischen 20.00 und 23.00 Uhr festgestellt worden (ZERBE, 1998). „Stärkere Tiere“ verweilten infolge der ständigen „Eigenbelohnungen“ häufiger und länger im Tränkestand und erwarben einen höheren Anspruch an „Spielsaugen“. Die sogenannten Kontrollbesuche (Saugen ohne Milchabruf) waren an allen Abrufzeitpunkten häufiger als die Besuche mit Milchabruf, sie waren überwiegend vor diesen zu beobachten, und ihre Häufigkeit nahm im Verlaufe des Tages an den Abrufzeitpunkten zu (ZERBE, 1998). Signifikant längere ZDA der Kälber aus HbG3 im Alter von 5 LT sind auf die größere körperliche Fitness dieser Tiere zurückzuführen. Längere ZDR der Kälber in HbG1 und HbG3 können unterschiedliche Ursachen haben. Bei Kälbern der HbG1 sind die zeitiger beginnende Ermüdung und physische Erschöpfung einzelner Tiere in Erwägung zu ziehen. Körperliches Training und Adaptation der Tiere führten zu einer Zunahme der mittleren ZDA und GZA und auch der ZDR und zu einer Abnahme der mittleren GZR bis zum Alter von 40 LT (Abb. 2 und 3).
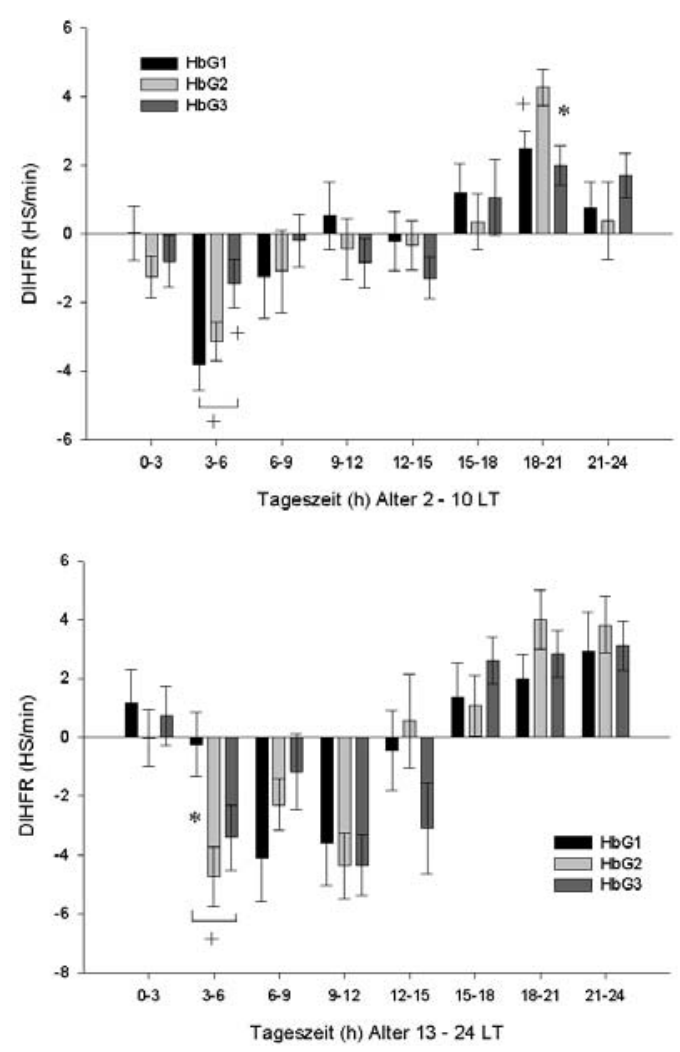

Abb. 6: Abweichung der Herzfrequenz während der Ruheperioden vom individuellen Mittelwert der Tagesaufzeichnung bei einem Alter der Milchrindkälber von 2 bis 10 LT (oberer Teil) und von 13 bis 24 LT (unterer Teil), weiter wie bei Abb. 1 (Deviation of heart rate from the mean of individual daily recording at resting times in calves of 2 to 10 days of life age (top) and of of 13 to 24 days of life age (bottom))

Rhythmen von HF, Blutdruck und Körpertemperatur, die mit den Wachzuständen (Arousal, Vigilanz) und Aktivitätsperioden der Tiere konform gehen (MATSUI und SUGANO, 1989; SATO et al., 1995; YAMAYA et al., 1994), sind durch endogene Oscillatoren bedingt (MISTLBERGER, 1994 , Ratte; MOHR und KRZYWANEK 
1995, Schafe; SATO et al. 1995, Kaninchen und Ratten) und können durch exogene Stimulierungen (Licht, Temperatur, betriebliche Ereignisse, soziale Beziehungen als Zeitgeber) synchronisiert werden. Eine Synchronisierung ist bereits beim Fetus infolge der placentären Passierbarkeit des Melatonin und der gleichartigen rhythmischen Variationen der Melatoninkonzentration beim Muttertier und bei den Nachkommen (Schaf: McMILLEN et al., 1995) sehr wahrscheinlich und bestätigt sich an der gut ausgeprägten Rhythmik der HFR der Kälber bei einem Alter von 5 LT (Abb. 6). Ein höheres Niveau der HF war beim Stehen der Tiere gegenüber dem Liegen (Saugkälber der Mutterkuhhaltung: LEHR, 1997; Tränkkälber: STEINHARDT und THIELSCHER, 2002) festgestellt worden. Größere Steigerungen der HF im Tagesverlauf in Beziehung zu den MAT-Abrufzeiten (Abb. 2) sind ein Ausdruck gesteigerter sympathischer nervaler Aktivität in Verbindung mit der Nahrungsaufnahme bei Jungtieren (BLOOM et al., 1975; BOWMAN et al. 1997, ERMGASSEN, 1996) und auch ein Ausdruck der motorischen und lokomotorischen Aktivität (Laufen, Springen).
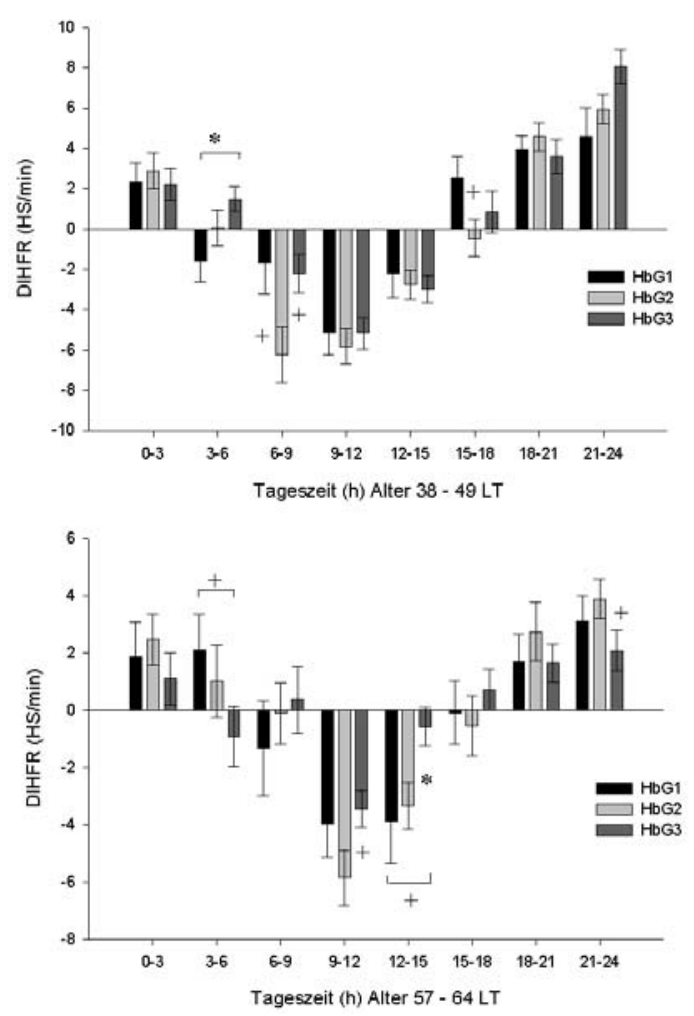

Abb. 7: Abweichung der Herzfrequenz während der Ruheperioden vom individuellen Mittelwert der Tagesaufzeichnung bei einem Alter der Milchrindkälber von 38 bis 49 LT (oberer Teil) und von 57 bis 64 LT (unterer Teil), weiter wie bei Abb. 1 (Deviation of heart rate from the mean of indivial daily recording at resting times in calves of 38 days of life age (top) and of 57 to 64 days of life age (bottom))

Die mit dem Tränken und der Kraftfutteraufnahme verbundenen Aktivitäten der Tiere am Automaten und die Geräusche können in Gruppenhaltungen als Zeitgeber wirken, in Abhängigkeit von Alter, Entwicklungsqualität und Nahrungsverfügbarkeit der Kälber eine Aktivierung eines größeren Anteils der Tiere und damit Synchronisierungseffekte zur Folge haben und auch das Spielverhalten der Tiere überlagern. Die unterschiedlichen Änderungen der Rhythmen der HF (Abb. 4 bis 7) bei Kälbern der HbG1, HbG2 und HbG3 sind in diesem Zusammenhang einzuschätzen. Kälber der HbG1 und HbG2 wiesen in den frühen Morgenstunden (03.00 bis 06.00 Uhr), diejenigen der 
HbG2 auch in den späten Abendstunden (18.00 bis 24.00 Uhr) größere Steigerungen der HFA auf (Abb. 4 und 5). Gewöhnung und vermehrte Festfutteraufnahme der Kälber können dazu beigetragen haben, dass die häufigen Wechsel von Ruhe und Aktivität in den ersten Lebenswochen geringer und die ZDR bei einem Alter von 40 LT signifikant länger wurden. Die Entwicklung sozialer Beziehungen der Kälber unter diesen Bedingungen ist bisher nicht ausreichend einzuschätzen. Angaben über das Spielverhalten der Kälber bei beständig wechselnder Gruppenzusammensetzung und über dessen gehäuftes Vorkommen an spezifischen Zeitpunkten im Tagesverlauf oder in Abhängigkeit von anderen Verhaltensweisen wie Nahrungsaufnahme und Körperschutzreaktionen sind kaum vorhanden. Die Hauptkomponenten des Spielverhaltens wie lokomotorisches Spielen in Gruppen, auch paralleles Spielen ohne physischen Kontakt genannt, spielerisches Kämpfen von zwei oder mehr Kälbern, spielerisches Aufspringen und Bodenspiel (BYERS, 1984) haben unterschiedliche Zeitanteile im Verlaufe der Aufzucht. Während das lokomotorische Spielen in Gruppen mehr bei jüngeren Kälbern zu beobachten ist, nehmen spielerisches Kämpfen, Aufspringen und Bodenspiel bei älteren Kälbern zu. Der Grad des lokomotorischen Spielens und dessen Synchronie verringerten sich über die Wochen bei mutterlos aufgezogenen Kälbern (Fütterung 2 mal pro Tag aus Eimern), das spielerische Kämpfen, in welches männliche Tiere mehr einbezogen waren, jedoch nicht (JENSEN et al., 1998; JENSEN und KYHN, 2000).

Die mit Hilfe der kontinuierlichen Herzfrequenzmessung an Kälbergruppen mit unterschiedlichem Hämoglobingehalt des Blutes an verschiedenen Alterspunkten vorgenommene Charakterisierung der Herzfrequenzkennwerte ermöglichten eine Einschätzung des Effektes der Entwicklungsqualität auf die Rhythmizität von Funktionsabläufen und auch von deren Änderungen infolge des Wachstums sowie der Anpassung der Kälber an die Haltungsbedingungen. Intensivere Messungen an speziellen Tiergruppen sind erforderlich, um die Anpassungsvorgänge der Kälber und die Zusammenhänge dieser Erscheinungen mit der Frequenz von direkten und indirekten Verlusten in der Aufzucht und auch Effekte der Anwendung geeigneter supplementierter Futtermittel genauer charakterisieren zu können.

\section{Literatur}

BLOOM, S.R.; EDWARDS, A.V.; HARDY, R.N.; MALINOWSKA, K.W.; SILVER, M.:

Cardiovascular and endocrine responses to feeding in the young calf. J. Physiol. Lond. 253 (1975), 135155

BOWMAN, E. C. J.; RODERICK, G. P.; BLOOM, S. R.; EDWARDS, A.V.:

Role of adrenoceptors in the hypertensive response to feeding in the conscious calf. Am. J. Physiol. 272 (Regulatory Integrative Comp. Physiol. 41) (1997), R607-R614

BYERS, J.A.:

Play in ungulates. In: SMITH, P. K. (ed.): Play in Animals and Humans. Basil Blackwell Publ. Lm. Pt. 1 (1984), 43-65

ERMGASSEN, K.:

Untersuchungen zur Herzfrequenz und zu klinischen Vitalitätsparametern bei Kälbern in Beziehung zu Tragzeit, Geburtsverlauf, Geschlecht und Rasse. Univ. Leipzig, Vet. med. Diss., 1996

JENSEN, M.B.; KYHN; R.:

Play behaviour in group-housed dairy calves, the effect of space allowance. Appl. Anim. Behav. Sci. 67 (2000), 35-46

JENSEN, M.B.; VESTERGAARD, K.S.; KROHN, C.C.:

Play behaviour in domestic calves kept in pens: the effect of social contact and space allowance. Appl. Anim. Behav. Sci. 56 (1998), 97-108 
LEHR, A.:

Verhaltensphysiologische Reaktionen bei Mutterkühen und ihren Kälbern im peripartalen Zeitraum. 3. Trenthorster Kolloquium 5. und 6. Dezember 1996. Landbauforschung Völkenrode, Sonderheft 177 (1997), 138-148

MATSUI, K.; SUGANO, S.:

Relation of intrinsic heart rate and autonomic nervous tone to resting heart rate in the young and the adult of various domestic animals. - Jpn. J. Vet. Sci. 51 (1989), 29-34

McMILLEN, I.C.; HOUGHTON, D.C.; YOUNG, I.R. :

Melatonin and the development of circadian and seasonal rhythmicity. J. Reprod. Fertil., Suppl. 49 (1995), 137-146

MISTLBERGER, R. E.:

Circadian food-anticipatory activity: formal models and physiological mechanisms. Neuroscience and Biobehavioural Rev. 18 (2) (1994), 171-195

MOHR, E. G.; KRZYWANEK, H.:

Endogenous oscillator and regulatory mechanisms of body temperature in sheep. Physiol. Behav. 57 (1995), 339-347

SATO, K.; CHATANI, F.; SATO, S.:

Circadian and short-term variabilities in blood pressure and heart rate measured by telemetry in rabbits and rats. J. Autonom. Nerv. Syst. 54 (1995), 234-246

STEINHARDT, M.; THIELSCHER, H.-H.; ERMGASSEN, K.; LEHR, A.:

Langzeitmessungen in entwicklungs- und verhaltensphysiologischen Untersuchungen bei landwirtschaftlichen Nutztieren am Beispiel der Herzschlagfrequenz. Schriftenreihe des Forschungsinstitutes für die Biologie landwirtschaftlicher Nutztiere (FBN), Heft 9 (1997), 47-70

STEINHARDT, M.; THIELSCHER, H.-H. :

Tiergerechte Haltung und physiologische Funktionen von Tieren. Entwicklungsqualität von Milchrindkälbern nach der Geburt und frühe postnatale Adaptation der Tiere in Gruppenhaltung mit Tränkeautomatenfütterung. Tierärztl. Umschau 55 (2000a) 4, 189-198

STEINHARDT, M.; THIELSCHER, H.-H.:

Wachstum und Entwicklungsqualität von Milchrindkälbern in Gruppenhaltung mit Tränkeautomatenfütterung. Physiologische Variablen und deren Änderungen in spezifischen Altersperioden. Arch. Tierz., Dummerstorf 43 (2000b) 1, 27-44

STEINHARDT,M.; THIELSCHER, H.-H. :

Herzfrequenz, Aktivitäts- und Ruhezeiten sowie Wachstumsleistung von in Gruppen mit Tränkeautomantenfütterung gehaltenen Milchrindkälbern während der Aufzucht im Winter und Frühjahr. Arch. Tierz., Dummerstorf 45 (2002a) 4, 359-373

STEINHARDT, M.; THIELSCHER, H.-H.:

Hämatologische Variablen und Plasmacortisol bei Milchrindern in Laufstallhaltung nach der Kalbung und bei deren Kälbern. Effekte einiger konstanter und variabler Faktoren. Arch. Tierz., Dummerstorf 45 (2002b) 5, 451-464

WILSON, L.L.; SMITH, J.L.; SMITH, D.L.; SWANSON, D.L.; DRAKE, T.R.; WOLFGANG, D.R.; WHEELER, E.F. :

Characteristics of veal calves upon arrivel, at 28 and 84 days, and at end of the production cycle. J. Dairy Sci. 83 (2000), 843-854

YAMAYA, Y.; KUBO, K.; AMADA, A.:

Diurnal rhythms of R-R interval and R-R interval variability in the young thoroughbred horse. J. Equine Sci. 5 (1994), 83-86

ZERBE, F.:

Einsatz von Tränkeautomaten in der Gruppenhaltung von Aufzuchtkälbern unter besonderer Berücksichtigung des Saug- und Futteraufnahmeverhaltens. Hannover, Vet. Med. Diss., 1998

Eingegangen: 08.08.2002

Akzeptiert: 26.09.2002

Anschrift der Verfasser

Dr. habil. MARTIN STEINHARDT, Dr. HANS - HERMANN THIELSCHER

Bundesforschungsanstalt für Landwirtschaft (FAL), Institut für Tierzucht und Tierverhalten

D-23847 Westerau 